\title{
Knockdown of PARP6 or survivin promotes cell apoptosis and inhibits cell invasion of colorectal adenocarcinoma cells
}

\author{
HAIPENG WANG, SHENGGUO LI, XISHUN LUO, ZHIKE SONG, XIANGKAI LONG and XIJIA ZHU \\ Department of Gastrointestinal Surgery, The Second Affiliated Hospital of Guilin Medical University, \\ Lingui, Guilin, Guangxi Zhuang Autonomous Region, Guangxi 541100, P.R. China
}

Received June 6, 2016; Accepted July 14, 2016

DOI: 10.3892/or.2017.5441

\begin{abstract}
Colorectal adenocarcinoma is the third most common cancer worldwide. PARP6, a novel member of the poly(ADP-ribose) polymerases (PARPs) and survivin, a member of the family of inhibitor of apoptosis (IAP) proteins are associated with a poor prognosis in various types of cancers. However, limited evidence exists regarding the interaction between PARP6 and survivin in colorectal adenocarcinoma. In the present study, we used the paired samples of 20 patients with colorectal adenocarcinoma to detect the expression of PARP6 and survivin in both tumor and adjacent normal colorectal mucosa. Their interaction and roles in cell viability, cell cycle, cell apoptosis and cell invasion were further investigated. Our results showed that both PARP6 and survivin exhibited higher expression in colorectal adenocarcinoma tissues and SW620 cells when compared with levels in adjacent non-tumor tissues and a normal colon cell line FHC. Co-immunoprecipitation assay showed that a significant correlation existed between PARP6 and survivin. We also showed that sole treatment of PARP6 siRNA or survivin siRNA partially inhibited the cell survival and invasion, induced cell G0/G1 arrest, and cell apoptosis at the early and late stages. The combined treatment of PARP6 siRNA and survivin siRNA suppressed the cell survival and cell invasion, further induced cell cycle phase G0/G1 arrest, and cell apoptosis at the early and late stages. Taken together, knockdown of PARP6 or survivin promotes cell apoptosis and inhibits the cell invasion of colorectal adenocarcinoma cells. A significant correlation exists between PARP6 and survivin, and both are promising targets for the development of new strategies for the diagnosis and treatment of advanced or metastatic colorectal adenocarcinoma.
\end{abstract}

Correspondence to: Dr Xijia Zhu, Department of Gastrointestinal Surgery, The Second Affiliated Hospital of Guilin Medical University, 212 Remin Road, Lingui, Guilin, Guangxi Zhuang Autonomous Region, Guangxi 541100, P.R. China

E-mail: zhuxijia2005@163.com

Key words: colorectal adenocarcinoma, poly(ADP-ribose) polymerases, PARP6, survivin, co-immunoprecipitation

\section{Introduction}

Colorectal adenocarcinoma is one of the most common malignant neoplasms (1). Although developments in the diagnosis and surgical treatment techniques in combination with neoadjuvant radiochemotherapy have improved the outcome of colorectal adenocarcinoma patients with a 5-year survival rate of $90 \%$, the 5 -year survival rate is only $12 \%$ for patients with metastatic colorectal adenocarcinoma (2). Recent studies provide increasing evidence that the cause of colorectal adenocarcinoma involves genetic and epigenetic alterations, morever, colorectal adenocarcinoma cells acquire invasive and metastatic capacities through the tumor microenvironment (3). Furthermore, investigations indicate that the tumor microenvironment not only enhances cancer metastasis, but also confers resistance to chemotherapy (4). Thus, it is imperative to elucidate the roles of proteases in the tumor microenvironment in order to achieve effective therapy, particularly against advanced and metastatic colorectal adenocarcinoma.

Poly(ADP-ribose) metabolism has an important biological function that mediates post-translational protein modification in the process of poly-ADP-glycosylation $(5,6)$. It can maintain genomic stability, regulate the transcriptional level and energy metabolism, and modify cell cycle progression and cell death (7). Poly(ADP-ribose) polymerases (PARPs) are a family of proteases, including PARP1-4, PARP $\alpha \alpha$, PARP5 $\beta$ and PARP6-16 (8). They play important roles in physiological and pathophysiological processes. Recently, it was shown that PARP6 likely encodes tumor suppressors, and an increase in its methylation was associated with a poor prognosis of various cancer, including hepatoblastoma, colorectal adenocarcinoma (9), breast (10), pancreatic (11), breast (12) and colorectal cancer (13). Among the PARPs, PARP1 is the member which has been studied most extensively. and was found to promote tumor angiogenesis (7). However, the roles of the new member PARP6 in cancer progressions and metastasis remain unelucidated.

PARP6 is located on chromosome 15q23 (8). It was demonstrated that overexpression of PARP6 suppressed the cell growth of HeLa cells (14). PARP6 was found to be negatively correlated with the Ki-67 proliferation index, and may be a marker for better prognosis in colorectal adenocarcinoma $(13,14)$. A recent study demonstrated that PAPR6 inhibited colony formation, invasion and cell proliferation in 
human colorectal adenocarcinoma cell line SW480 (13), and indicated an interaction between the overexpression of PARP6 and the downregulation of survivin. The authors reported an inverse correlation between PARP6 and survivin expression in colorectal adenocarcinoma tissues as confirmed by immunohistochemistry using tumor tissue and normal colonic mucosa (13).

Survivin, a member of the family of inhibitor of apoptosis (IAP) proteins, takes part in the inhibition of cell apoptosis, which is also related to the poor prognosis of various human cancers, including colorectal adenocarcinoma $(13,15-19)$. The survivin mRNA-circulating tumor cells are associated with prostate cancer metastasis (20). Suppression of survivin induces mitochondrial-mediated apoptosis in gastric cancer cells $(21,22)$. In addition, survivin is involved in the cell cycle arrest of colorectal cancer cells (23). Increased apoptosis by a survivin inhibitor is considered as an effective treatment for colon cancer (24). However, limited evidence exists regarding the interaction between PARP6 and survivin in cell proliferation, the cell cycle and cell apoptosis.

In the present study, we used paired samples from 20 patients with colorectal adenocarcinoma. Expression of PARP6 and survivin in both tumor tissues and adjacent normal colorectal mucosa was assessed. In addition, their interaction and roles in cell viability, cell cycle and cell apoptosis were further investigated.

\section{Materials and methods}

Patients and cell culture. Twenty patients with colorectal cancer were selected. Patients who had received chemotherapy or a family history of polypus or IBD before surgery were excluded. All samples were fixed in neutral buffered formalin $10 \%$ and paraffin blocked. All cut sections from both tumor and adjacent normal colorectal mucosa were selected, and immunohistochemical staining was performed for expression analyses. All patients who participated in the present study provided written informed consent. The experimental protocol for human subjects was approved by the Ethics Committee of The Second Affiliated Hospital of Guilin Medical University.

The human colorectal adenocarcinoma SW620 cells and normal colon cell line FHC were incubated in complete Leibovitz's L-15 medium with tetracycline-free $10 \%$ fetal bovine serum (FBS) (Gibco, Thermo Fisher Scientific), $100 \mathrm{U} /$ $\mathrm{ml}$ penicillin and $100 \mu \mathrm{g} / \mathrm{ml}$ streptomycin. SW620 cells were transfected with PARP6 siRNA or survivin siRNA using DharmaFECT reagent (Life Technologies, Grand Island, NY, USA) according to the manufacturer's instructions. The siRNA sequences are listed in Table I.

Quantitative real-time polymerase chain reaction ( $q R T-P C R)$. After six days of transfection, total RNA was isolated and the transcription levels of PARP6 and survivin were determined by real-time reverse transcriptase polymerase chain reaction (25). The primer pairs were: TTTGAGCCTTATCCC TCTGTG (sense) and TGGGTCATCTCCCGAATAGA (antisense) for PARP6; TTTGAGGAAACTGCGGAGAA (sense) and GGTGGCACCAGGGAATAAA (antisense) for survivin; and primers for $\beta$-actin ATCGTGCGTGACATTAAGG AGAAG (sense) and AGGAAGGAAGGCTGGAAGAGTG
Table I. The siRNA sequences.

\begin{tabular}{|c|c|c|c|}
\hline & Position & & Sequences \\
\hline \multirow{3}{*}{$\begin{array}{l}\text { PARP6 } \\
\text { siRNA }\end{array}$} & $1808-1830$ & S5' & GGCGAUGCCAACAUUAAUAdTdT \\
\hline & & mRNA & TGGGCGATGCCAACATTAATACT \\
\hline & & AS $3^{\prime}$ & TdTdCCGCUACGGUUGUAAUUAU \\
\hline \multirow{3}{*}{$\begin{array}{l}\text { Survivin } \\
\text { siRNA }\end{array}$} & $268-290$ & S5' & GAAGCAGUUUGAAGAAUUAdTdT \\
\hline & & mRNA & AAGAAGCAGTTTGAAGAATTAAC \\
\hline & & AS $3^{\prime}$ & TdTdCUUCGUCAAACUUCUUAAU \\
\hline
\end{tabular}

(antisense). The final result was expressed as $\log 2$ of $2^{-\Delta \mathrm{CT}}$ calculation, and the levels of mRNA were normalized to $\beta$-actin (25).

Western blotting. Western blotting was performed six days post-transfection. Proteins were extracted from the cells, and then a total of $30 \mu \mathrm{g}$ proteins was separated using $10 \%$ SDS-PAGE and transferred to polyvinylidene difluoride (PVDF) membranes. The membranes were incubated with PARP6 $(1: 1,000)$ and survivin antibodies $(1: 1,000)$ (both from Santa Cruz Biotechnology, Inc., Santa Cruz, CA, USA) for $1 \mathrm{~h}$ at room temperature (RT) followed by incubation with the secondary antibody for $1 \mathrm{~h}$. Visualization of the bands was performed using an ECL kit (Beyotime, Shanghai, China). Quantification of protein bands was performed using Gel-Pro Analyzer software (Media Cybernetics, Inc., Rockville, MD, USA).

Co-immunoprecipitation (Co-IP) of PARP6 and survivin. Co-IP of PARP6 and survivin was performed. Briefly, the cells were homogenized, supernatants collected and aliquots were separated for protein determination and immunoblotting. Survivin antibody or IgG was coupled to SiezeX beads following the kit protocol (Pierce, Rockford, IL, USA). Proteins were eluted, separated on 12-15\% SDS-PAGE, and then transferred to nitrocellulose for PARP6 immunoblotting.

Immunohistochemistry and immunofluorescence staining. Immunohistochemistry (IHC) for the detection of PARP6 and survivin was carried out using antibodies for PARP6 $(1: 1,000)$ and survivin $(1: 1,000)$ and the EnVision detection kit (Dako, Carpinteria, CA, USA). The standard procedures for IHC were performed as described in a previous study (26).

Expression and distribution of PARP6 and survivin in cells were determined by indirect immunofluorescence microscopy, as previously described (27). Briefly, the cells were fixed with $3.5 \%$ PFA in phosphate-buffered saline (PBS) for $10 \mathrm{~min}$ at RT and permeated and blocked with $0.1 \%$ Triton X-100 and $5 \%$ FCS in PBS for $30 \mathrm{~min}$. The fixed cells were washed and incubated for $1 \mathrm{~h}$ with PARP6 or survivin antibody, and then washed and incubated with secondary antibodies for $30 \mathrm{~min}$, and observed under a fluorescence microscopy.

CCK-8 assay. Cells were seeded in 96-well plates at a density of $3 \times 10^{4}$ cells $/ 0.1 \mathrm{ml}$ and were cultured for various days. Then, $10 \mu 1$ of Cell Counting Kit-8 (CCK-8) solution (KeyGen Biotech, Nanjing, China) was added to each well of the plate. 


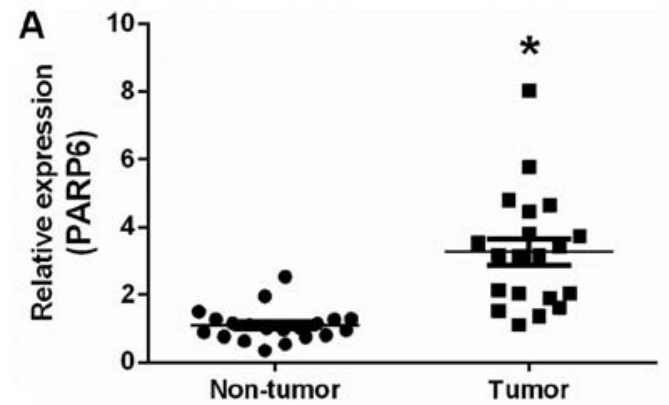

C

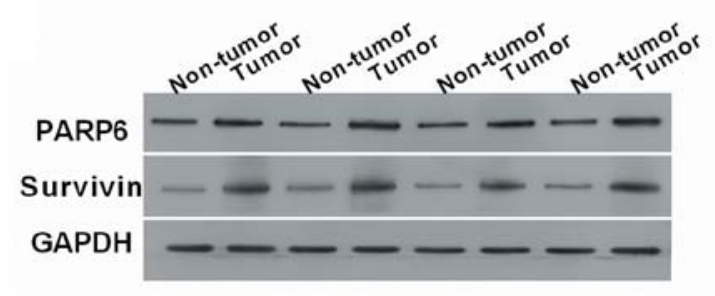

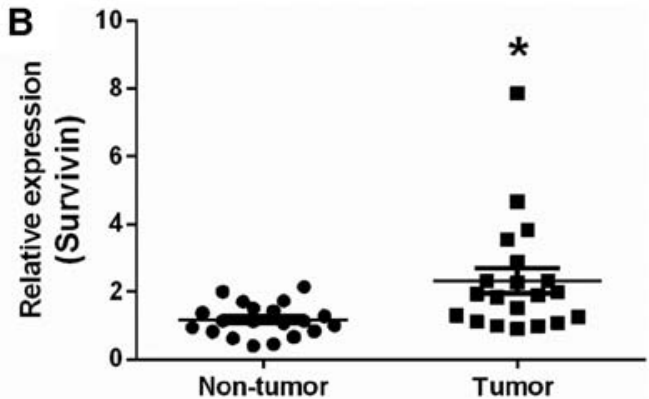

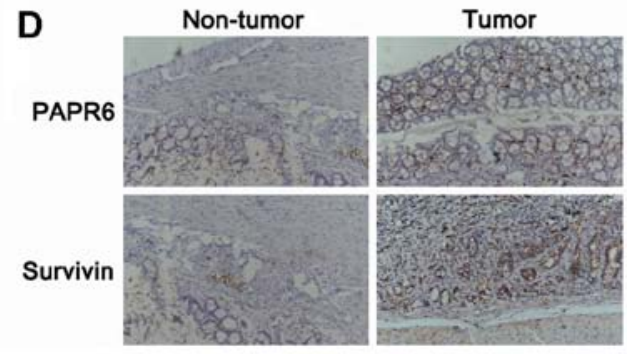

Figure 1. Expressions of PARP6 and survivin in human colorectal cancer (tumor) and adjacent normal tissues (non-tumor). Relative mRNA expression of (A) PARP6 and (B) survivin as detected by qRT-PCR. (C) Western blot analysis of the expression of PARP6 and survivin. (D) Immunohistochemical analysis of PARP6 and survivin in non-tumor and colorectal cancer tissues; ${ }^{*} \mathrm{P}<0.05$ vs. non-tumor tissues.

After a 4-h incubation, the plates were analyzed at $450 \mathrm{~nm}$. All values are expressed as the means \pm SD of at least three wells and at least three independent experiments.

Flow cytometry. After six days of transfection, cell apoptosis, and cell cycle distribution were analyzed by flow cytometry. Cell apoptosis was detected using FITC-conjugated Annexin V and propidium iodide (PI; Beyotime). Cell cycle distribution was detected using the Cell Cycle Staining kit (Multi Sciences, Hangzhou, China) according to the manufacturer's instructions.

Cell invasion assay. After six days of transfection, cell invasion was examined using a reconstituted extracellular matrix membrane (BD Biosciences, San Jose, CA, USA). Cells suspended in serum-free media at a concentration of $3 \times 10^{4}$ cells $/ 0.5 \mathrm{ml}$ were placed in the upper chambers, and complete medium containing $10 \%$ FBS and $1 \%$ FBS was added to the lower chambers. After 18-24 h, the chambers were fixed with methanol for $30 \mathrm{~min}$ and stained with crystal violet for an additional $30 \mathrm{~min}$. The migrated cells were imaged.

Statistical analysis. All experiments were at least repeated in triplicate. All data were analyzed using SPSS by the Student's t-test and expressed as the mean \pm SD. A p-value $<0.05$ was considered as statistically significant.

\section{Results}

Expression of PARP6 and survivin in human colorectal cancer tissues. We examined the expression levels of PARP6 and survivin in human colorectal cancer (tumor) and adjacent normal tissue (non-tumor) (Fig. 1). The results showed that PARP6 mRNA (Fig. 1A) and survivin mRNA (Fig. 1B) were significantly upregulated in the tumor tissues. At the protein level, PARP6 and survivin were upregulated in the tumor tissues (Fig. 1C), compared to these levels in the non-tumor tissues. The upregulation of PARP6 and survivin was also validated by IHC (Fig. 1D). PARP6 was mostly expressed closed to the nucleus, while survivin was mostly expressed in the cytoplasm.

Expression of PARP6 and survivin in FHC and SW620 cells. We examined the expression of PARP6 and survivin in colorectal adenocarcinoma cell line SW620 and a normal colon cell line FHC (Fig. 2). The results showed that both PARP6 mRNA (Fig. 2A) and survivin mRNA (Fig. 2B) were upregulated in the SW620 cells, compared with the mRNA levels in the FHC cells. Similar changes were also observed at the protein levels (Fig. 2C) and the immunostaining showed higher expression of PARP6 (Fig. 2D) and survivin (Fig. 2E) in the SW620 cells, compared with the levels in the FHC cells. Thus, PARP6 and survivin were upregulated in colorectal adenocarcinoma cells.

Correlation between PARP6 and survivin in colorectal adenocarcinoma. Silencing of PARP6 inhibited the expression of PARP6 mRNA, and silencing of survivin only slightly inhibited the expression of PARP6 (Fig. 3A). Silencing of survivin inhibited the expression of survivin mRNA, and silencing of PARP6 only slightly inhibited the expression of survivin (Fig. 3B). Similar results were shown at the protein levels (Fig. 3C). The combination of PARP6 siRNA and survivin siRNA inhibited the expression of PARP6 and survivin at both the mRNA and protein levels. We also examined the cell survival by CCK-8 assay (Fig. 3D). Silencing of PARP6 or survivin inhibited the cell survival of the SW620 cells. In addition, the combined treatment of PARP6 siRNA and survivin siRNA further inhibited the cell survival in the SW620 cells, suggesting that PARP6 and survivin play roles 


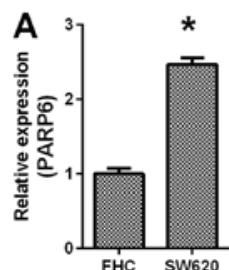

D
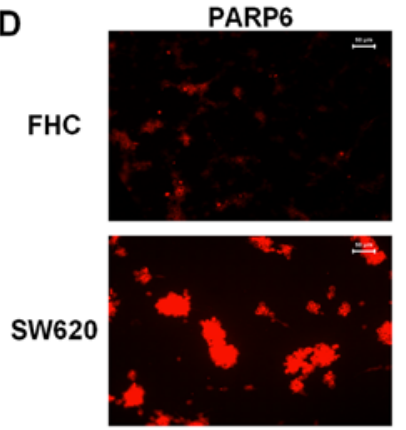

E

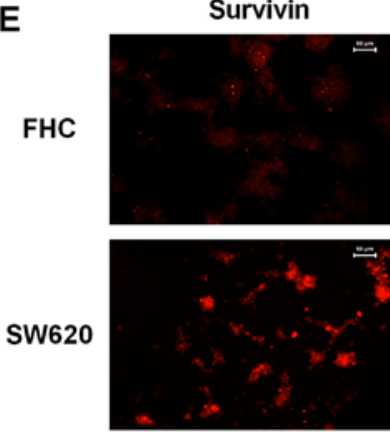

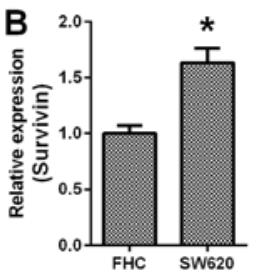

DAPI

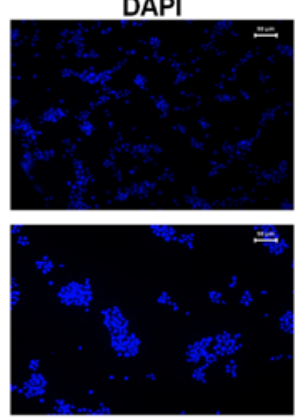

DAPI
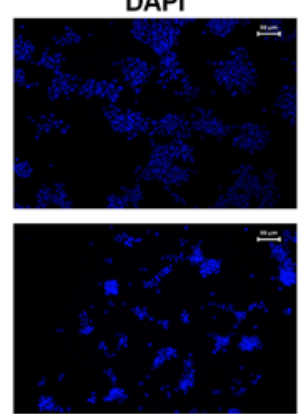

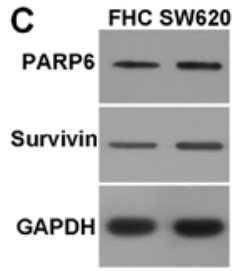

MERGE

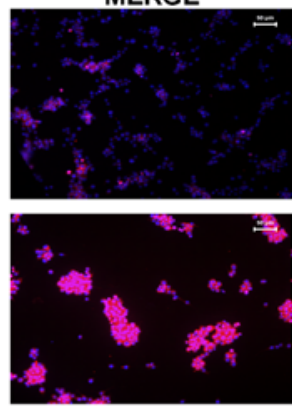

MERGE

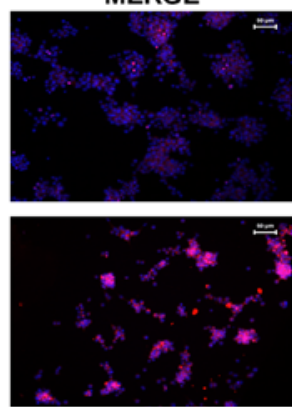

Figure 2. Expression of PARP6 and survivin in FHC and SW620 cells. Relative mRNA expression of (A) PARP6 and (B) survivin as determined by qRTPCR. (C) Protein expression levels were detected by western blotting. Immunostaining analysis of (D) PARP6 (red fluorescence) and (E) survivin (red fluorescence) in FHC and SW620 cells. Blue fluorescence represents nuclear staining; original magnification, $\mathrm{x} 400$; ${ }^{*} \mathrm{P}<0.05$ vs. the FHC cells.

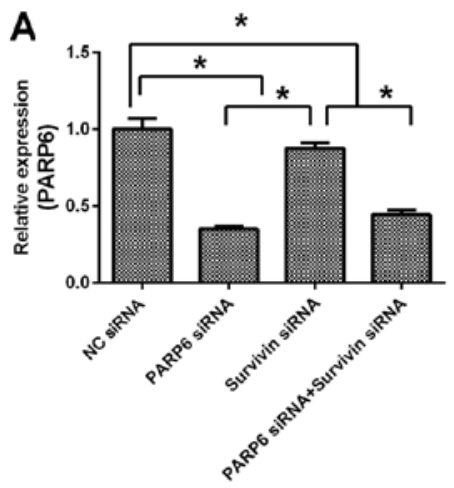

D

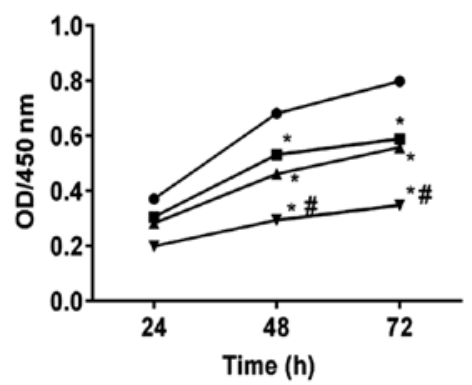

B

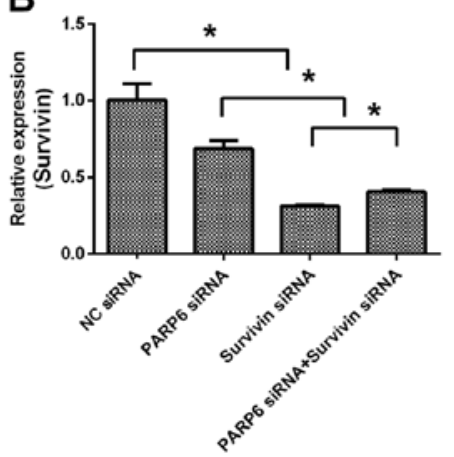

C

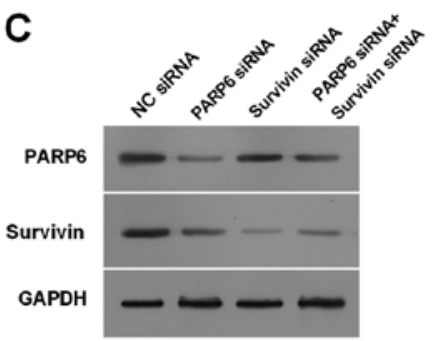

$\mathbf{E}$

$\rightarrow$ NC siRNA

$\rightarrow$ PARP6 siRna

- Survivin siRnA

* PARP6-Survivin siRNA

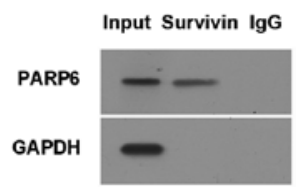

Figure 3. Interaction between PARP6 and survivin in SW620 cells. Effects of PARP6 siRNA, survivin siRNA, and PARP6 siRNA + survivin siRNA on the expression of PARP6 and survivin at the (A and B) mRNA and (C) protein levels; ${ }^{*} \mathrm{P}<0.05$. (D) CCK-8 assay was carried out to determine the cell viability in the PARP6 siRNA-, survivin siRNA-, and PARP6 siRNA + survivin siRNA-transfected SW620 cells; "P<0.05 vs. NC siRNA; "P $<0.05$ vs. PARP6 siRNA. (E) Co-IP detection verified the authenticity of the interaction between PARP6 and survivin in the SW620 cells. 
A
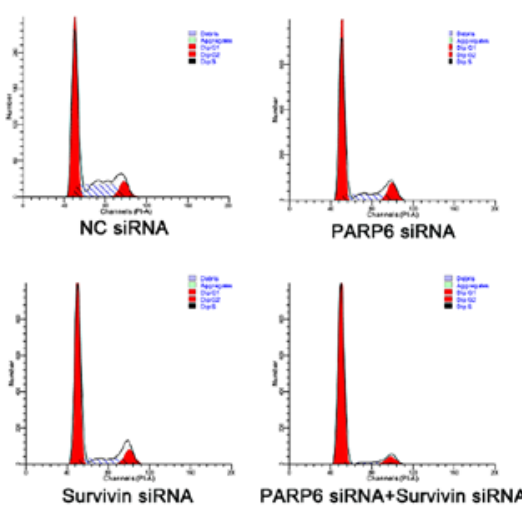

C
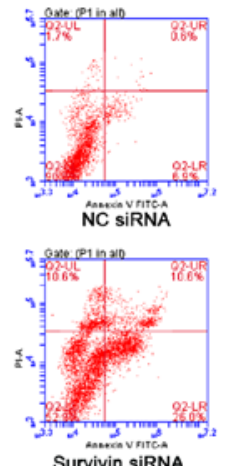
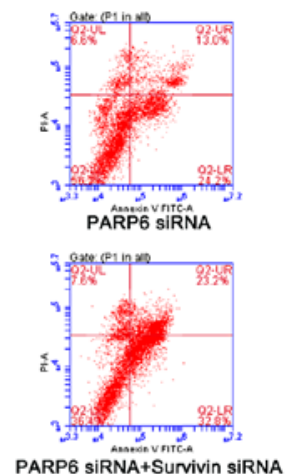

B
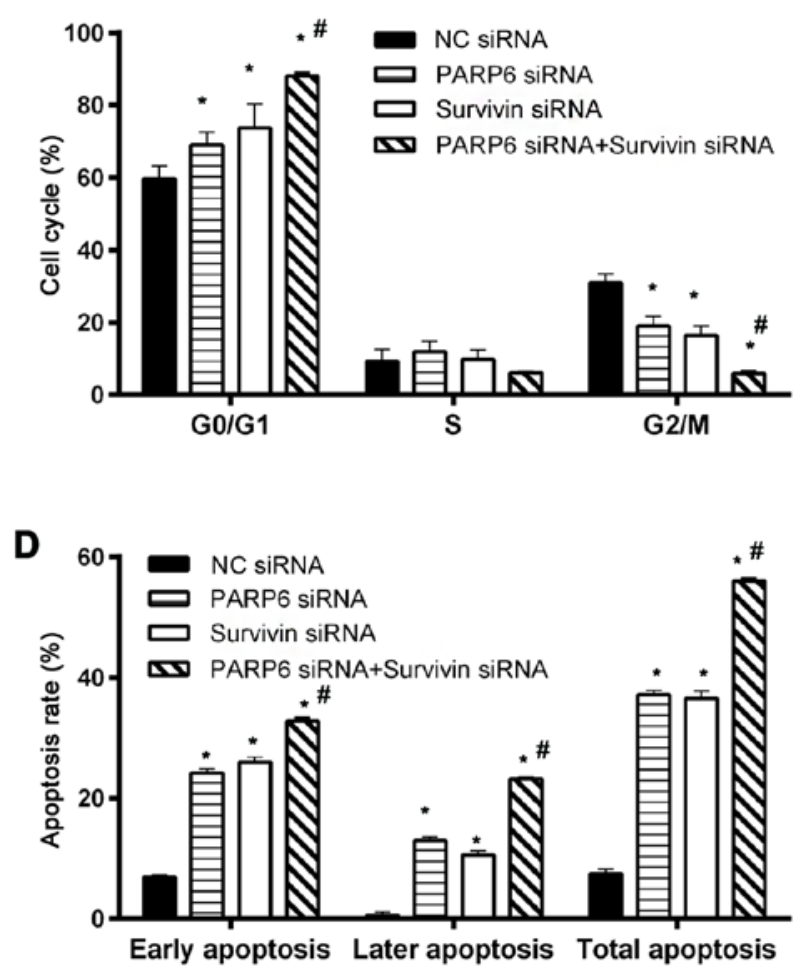

Figure 4. Flow cytometric analysis of the cell cycle and cell apoptosis in the PARP6 siRNA-, survivin siRNA- and PARP6 siRNA + survivin siRNA-transfected SW620 cells. (A) Cell cycle and (B) cell distribution in the G0/G1, S and G2/M phases in the transfected SW620 cells. (C) Cell apoptosis and (D) the percentage of apoptotic transfected SW620 cells; "P $<0.05$ vs. NC siRNA; "P $<0.05$ vs. PARP6 siRNA.

A

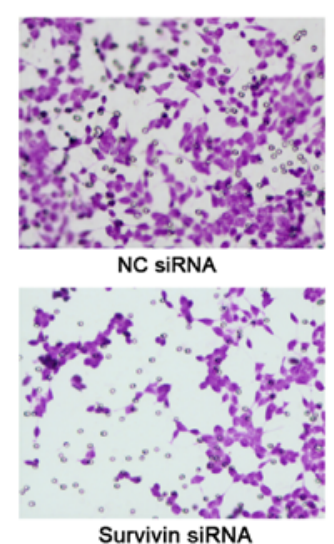

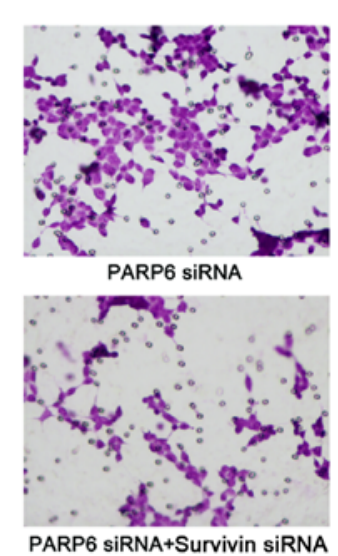

B

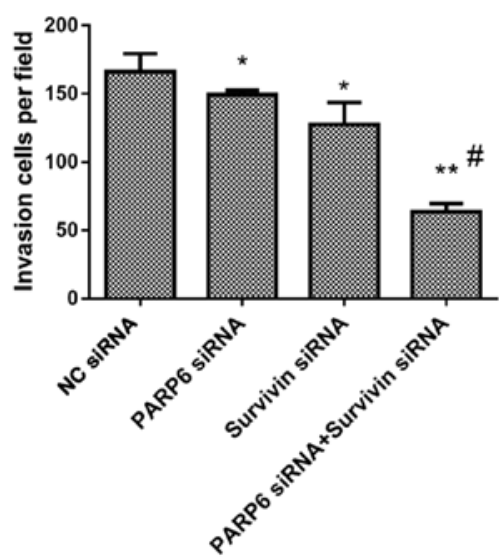

Figure 5. Cell invasion assay. (A) Transwell assay was carried out to assess the cell invasion in the PARP6 siRNA-, survivin siRNA-, and PARP6 siRNA + survivin siRNA-transfected SW620 cells. (B) The invasive cells in each group were quantified; ${ }^{*} \mathrm{P}<0.05$ vs. NC siRNA; ${ }^{* *} \mathrm{P}<0.01$ vs. NC siRNA; ${ }^{*} \mathrm{P}<0.05$ vs. PARP6 siRNA.

in the cell survival in different pathways. In order to detect the correlation between PARP6 and survivin in SW620 cells, Co-IP was used to identify the interactive protein (Fig. 3E). The results revealed that PAPR6 was present in the input and in the final homogenate collected after Co-IP, but no signal for PAPR6 was present in the IgG Co-IP lane. This confirmed that PARP6 interacts with survivin.

Cell cycle and cell apoptosis. Cell cycle and cell apoptosis were further assessed (Fig. 4). Silencing of PARP6 or survivin increased the percentage of cells in the G0/G1 phases and decreased the percentage of cells in the G2/M phase (Fig. 4A and B). In addition, treatment with the combination of PARP6 siRNA and survivin siRNA further increased the percentage of cells in the G0/G1 phases and decreased the cells in the G2/M phase. The cell cycle arrest in the G0/G1 phase was associated with changes in cell survival (Fig. 3D) and apoptosis (Fig. 4C and D). Silencing of PARP6 or survivin increased both early and late apoptosis. In addition, the silencing of PARP6 and survivin further 
enhanced the rate of early and late cell apoptosis, suggesting that PARP6 and survivin play roles in cell cycle and apoptosis in different pathways.

Cell invasion. Silencing of PARP6 or survivin decreased the invasion of the SW620 cells, and this was further attenuated by the combined silencing of PARP6 and survivin (Fig. 5), also suggesting that PARP6 and survivin play roles in cell invasion in different pathways.

\section{Discussion}

We demonstrated a correlation between PARP6 and survivin. These findings differ from a previous report (13). This is possible due to the different stage of the samples collected and the different cell model used. In the present study, we found that sole treatment of PARP6 siRNA or survivin siRNA partially inhibited cell survival and invasion, induced cell G0/ G1 arrest, and cell apoptosis in the early and late stages. The combined treatment of PARP6 siRNA and survivin siRNA further suppressed the cell survival, further induced cell cycle G0/G1 arrest, and cell apoptosis in the early and late stages. All of these results indicate that PARP6 and survivin play an important role in colorectal adenocarcinoma through a distinct pathway, which should be further investigated in the future.

PARPs are DNA-dependent nuclear enzymes. They regulate the interactions between protein and protein, and protein and DNA, by transferring negatively charged ADP-ribose moieties to protein substrates (28), playing important roles in physiological and pathophysiological processes. A total of 17 members of the PARP family have been identified including PARP1-4, PARP5 $\alpha$, PARP5 $\beta$ and PARP6-16 (8). Among the PARPs, PARP1 was found to promote tumor angiogenesis (7). PARP6 located on chromosome 15q23 (8), may be a marker for better prognosis of hepatoblastoma (9), breast $(10,12)$, pancreatic (11), and colorectal cancer (13). Overexpression of PARP6 was found to suppress the cell growth of HeLa cells, and is correlated with a decrease in the Ki-67 proliferation index $(13,14)$. In human colorectal adenocarcinoma cell line SW480, PAPR6 inhibited colony formation, invasion and cell proliferation (13). However, PARP6 was found to be downregulated in cases of colorectal cancer (13). This is inconsistent with our finding that PARP6 was overexpression and is located in the region close to the nuclear membrane in colorectal adenocarcinoma cells, compared to adjacent normal colorectal mucosa.

Survivin is also correlated with the poor prognosis of colorectal adenocarcinoma patients $(13,15-19)$. Survivin takes part in the cell apoptosis of gastric cancer cells $(21,22)$, and is involved in cell cycle arrest in colorectal cancer (23). Increased apoptosis by a survivin inhibitor is considered as an effective treatment for colon cancer (24). In the present study, we also demonstrated that survivin is overexpressed in colorectal adenocarcinoma, and is mostly present in the cytoplasm. Thus, PARP6 and survivin are located in distinct regions, and the CO-IP assay showed a significant correlation between PARP6 and survivin in the SW620 cells and colorectal adenocarcinoma tissues.

Our further investigation on cell survival, cell cycle and cell apoptosis further supported these findings. Knockdown of PARP6 inhibited the expression of survivin in part while knockdown of survivin only inhibited the expression of PARP6 in part. Sole knockdown of PARP6 or survivin partially inhibited the cell survival, induced cell cycle G0/G1 arrest, and cell apoptosis in the early and late stages. When both PARP6 and survivin were knocked down, the cell survival and cell invasion were further suppressed, and the cell cycle G0/ G1 arrest and cell apoptosis in the early and later stage were further enhanced. All of these results indicate that PARP6 and survivin play an important role in colorectal adenocarcinoma through a distinct pathway. This is inconsistent with the literature demonstrating there is an inverse correlation between PARP6 and survivin expression in colorectal adenocarcinoma tissues (13).

In conclusion, knockdown of PARP6 or survivin promoted cell apoptosis and inhibited cell invasion in colorectal adenocarcinoma. A significant correlation exists between PARP6 and survivin. They are promising targets for the development of new strategies for the diagnosis and treatment of advanced or metastatic colorectal adenocarcinoma.

\section{Acknowledgements}

The present study was supported by the China Guilin Scientific Research and Technological Development Project (no. 20140505-1).

\section{References}

1. Robbins AS, Siegel RL and Jemal A: Racial disparities in stage-specific colorectal cancer mortality rates from 1985 to 2008. J Clin Oncol 30: 401-405, 2012.

2. Bultman SJ: Interplay between diet, gut microbiota, epigenetic events, and colorectal cancer. Mol Nutr Food Res: May 3, 2016 (Epub ahead of print). doi: 10.1002/mnfr.201500902.

3. Itatani Y, Kawada K, Inamoto S, Yamamoto T, Ogawa R, Taketo MM and Sakai Y: The role of chemokines in promoting colorectal cancer invasion/metastasis. Int J Mol Sci 17: pii: E643, 2016.

4. Meads MB, Gatenby RA and Dalton WS: Environment-mediated drug resistance: A major contributor to minimal residual disease. Nat Rev Cancer 9: 665-674, 2009.

5. Kraus WL: Transcriptional control by PARP-1: Chromatin modulation, enhancer-binding, coregulation, and insulation. Curr Opin Cell Biol 20: 294-302, 2008.

6. Clayton C and Hotz HR: Post-transcriptional control of PARP gene expression. Mol Biochem Parasitol 77: 1-6, 1996.

7. Hassa PO and Hottiger MO: The diverse biological roles of mammalian PARPS, a small but powerful family of poly-ADPribose polymerases. Front Biosci 13: 3046-3082, 2008.

8. Hakmé A, Wong HK, Dantzer F and Schreiber V: The expanding field of poly(ADP-ribosyl)ation reactions. 'Protein Modifications: Beyond the Usual Suspects' Review Series. EMBO Rep 9: 1094-1100, 2008.

9. Honda S, Minato M, Suzuki H, Fujiyoshi M, Miyagi H, Haruta M, Kaneko Y, Hatanaka KC, Hiyama E, Kamijo T, et al: Clinical prognostic value of DNA methylation in hepatoblastoma: Four novel tumor suppressor candidates. Cancer Sci 107: 812-819, 2016.

10. Gonçalves A, Sabatier R, Charafe-Jauffret E, Gilabert M, Provansal M, Tarpin C, Extra JM, Viens P and Bertucci F: Triple-negative breast cancer: Histoclinical and molecular features, therapeutic management and perspectives. Bull Cancer 100: 453-464, 2013 (In French).

11. Porcelli L, Quatrale AE, Mantuano P, Leo MG, Silvestris N, Rolland JF, Carioggia E, Lioce M, Paradiso A and Azzariti A: Optimize radiochemotherapy in pancreatic cancer: PARP inhibitors a new therapeutic opportunity. Mol Oncol 7: 308-322, 2013. 
12. Salemi M, Galia A, Fraggetta F, La Corte C, Pepe P, La Vignera S, Improta G, Bosco P and Calogero AE: Poly (ADP-ribose) polymerase 1 protein expression in normal and neoplastic prostatic tissue. Eur J Histochem 57: e13, 2013.

13. Qi G, Kudo Y, Tang B, Liu T, Jin S, Liu J, Zuo X, Mi S, Shao W, Ma X, et al: PARP6 acts as a tumor suppressor via downregulating Survivin expression in colorectal cancer. Oncotarget 7: 18812-18824, 2016.

14. Tuncel H, Tanaka S, Oka S, Nakai S, Fukutomi R, Okamoto M, Ota T, Kaneko H, Tatsuka M and Shimamoto F: PARP6, a mono(ADP-ribosyl) transferase and a negative regulator of cell proliferation, is involved in colorectal cancer development. Int J Oncol 41: 2079-2086, 2012.

15. Fragni M, Bonini SA, Stabile A, Bodei S, Cristinelli L, Simeone C, Zani D, Spano PF, Berruti A, Memo M, et al: Inhibition of survivin is associated with zoledronic acid-induced apoptosis of prostate cancer cells. Anticancer Res 36: 913-920, 2016.

16. Wu J, Zhao S, Zhang J, Qu X, Jiang S, Zhong Z, Zhang F, Wong $\mathrm{Y}$ and Chen $\mathrm{H}$ : Over-expression of survivin is a factor responsible for differential responses of ovarian cancer cells to S-allylmercaptocysteine (SAMC). Exp Mol Pathol 100: 294-302, 2016.

17. Huang W, Mao Y, Zhan Y, Huang J, Wang X, Luo P, Li LI, Mo D, Liu Q, Xu H, et al: Prognostic implications of survivin and lung resistance protein in advanced non-small cell lung cancer treated with platinum-based chemotherapy. Oncol Lett 11: 723-730, 2016.

18. Ma WH, Liu YC, Xue ML, Zheng Z and Ge YL: Downregulation of survivin expression exerts antitumoral effects on mouse breast cancer cells in vitro and in vivo. Oncol Lett 11: 159-167, 2016.

19. Zhu J, Sun C, Wang L, Xu M, Zang Y, Zhou Y, Liu X, Tao W, Xue B, Shan Y, et al: Targeting survivin using a combination of miR 494 and survivin shRNA has synergistic effects on the suppression of prostate cancer growth. Mol Med Rep 13: 1602-1610, 2016.
20. Wang H, Yang M, Xu J, Zou B, Zhou Q, Bian J and Wang X: Survivin mRNA-circulating tumor cells are associated with prostate cancer metastasis. Tumour Biol 37: 723-727, 2016.

21. Yang B, Huang J, Liu H, Guo W and Li G: miR-335 directly, while miR-34a indirectly modulate survivin expression and regulate growth, apoptosis, and invasion of gastric cancer cells Tumour Biol 37: 1771-1779, 2016.

22. Wang TA, Zhang XD, Guo XY, Xian SL and Lu YF: 3-Bromopyruvate and sodium citrate target glycolysis, suppress survivin, and induce mitochondrial-mediated apoptosis in gastric cancer cells and inhibit gastric orthotopic transplantation tumor growth. Oncol Rep 35: 1287-1296, 2016.

23. Zhang B, Leng C, Wu C, Zhang Z, Dou L, Luo X, Zhang B and Chen X: Smad4 sensitizes colorectal cancer to 5-fluorouracil through cell cycle arrest by inhibiting the PI3K/Akt/CDC2/ survivin cascade. Oncol Rep 35: 1807-1815, 2016.

24. Li WL, Lee MR and Cho MY: The small molecule survivin inhibitor YM155 may be an effective treatment modality for colon cancer through increasing apoptosis. Biochem Biophys Res Commun 471: 309-314, 2016.

25. Steponaitis G, Skiriutè D, Kazlauskas A, Golubickaitė I, Stakaitis R, Tamašauskas A and Vaitkienè P: High CHI3LI expression is associated with glioma patient survival. Diagn Pathol 11: 42, 2016.

26. Qu Y, Gu C, Wang H, Chang K, Yang X, Zhou X, Dai B, Zhu Y, Shi G, Zhang H, et al: Diagnosis of adults Xp11.2 translocation renal cell carcinoma by immunohistochemistry and FISH assays: Clinicopathological data from ethnic Chinese population. Sci Rep 6: 21677, 2016

27. Lee WK and Kang JS: Modulation of apoptosis and differentiation by the treatment of sulfasalazine in rabbit articular chondrocytes. Toxicol Res 32: 115-121, 2016.

28. Schreiber V, Dantzer F, Ame JC and de Murcia G: Poly(ADP-ribose): Novel functions for an old molecule. Nat Rev Mol Cell Biol 7: 517-528, 2006. 